Organo- and

Biocatalysis

\title{
Organocatalytic C-H Hydroxylation
}

Key words

C-H hydroxylation

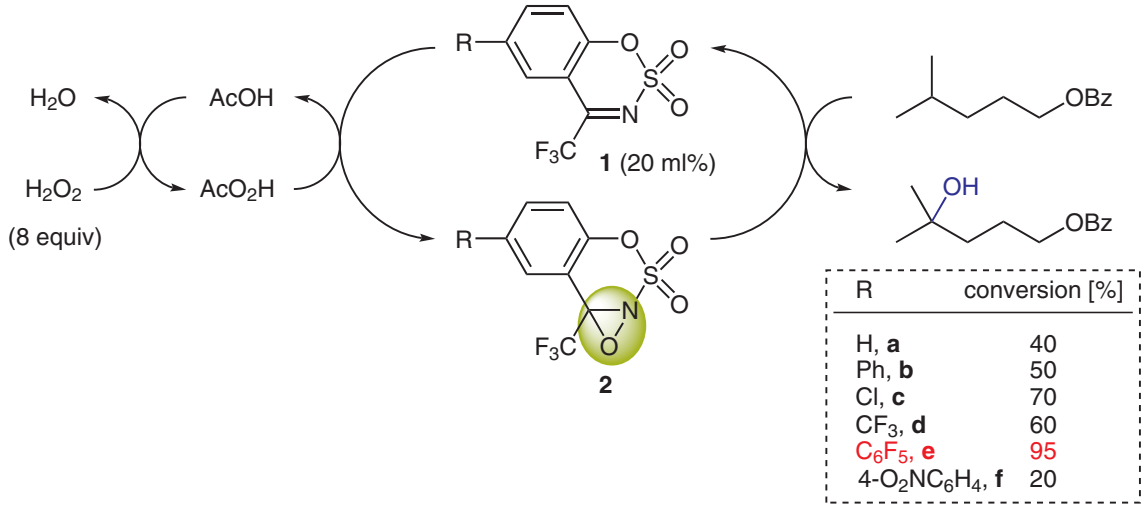

oxaziridines

hydrogen peroxide

hydrophobic

aggregation
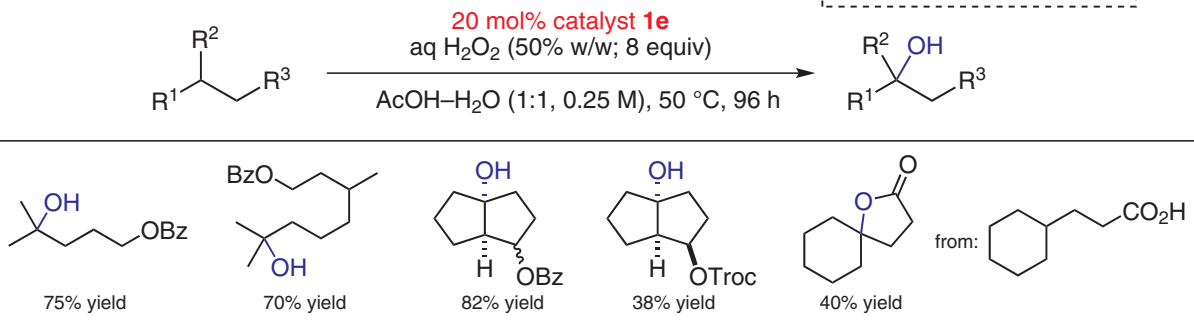

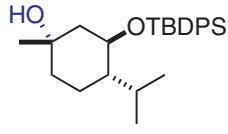

$34 \%$ yield
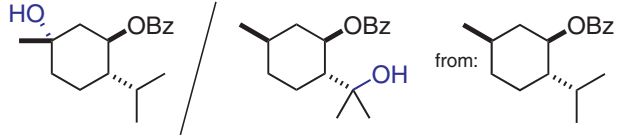

$47 \%$ yield $(\sim 1: 1)$
Significance: Based on the authors' previous work in this field (J. Am. Chem. Soc. 2005, 127, 15391) a second-generation oxaziridine 2-mediated catalytic process for $\mathrm{C}-\mathrm{H}$ hydroxylation is reported. The revised reaction protocol features the use of aqueous $\mathrm{H}_{2} \mathrm{O}_{2}$ in $\mathrm{AcOH}-\mathrm{H}_{2} \mathrm{O}$ (1:1), conditions which efficiently generate the reactive oxaziridine intermediate $\mathbf{2}$ in situ from the benzoxathiazine catalyst $\mathbf{1}$. Among all catalysts $\mathbf{1}$ tested, catalyst 1e $\left(R=\mathrm{C}_{6} \mathrm{~F}_{5}\right)$ turned out to be the most effective suggesting hydrophobic catalyst-substrate aggregation as proposed by the authors. Mechanistically, oxaziridine-mediated electrophilic $\mathrm{O}$-atom insertion into $\mathrm{C}-\mathrm{H}$ bonds likely proceeds in a concerted, asynchronous fashion.
Comment: Most catalytic $\mathrm{C}-\mathrm{H}$ bond hydroxylation processes rely on transition metal complexes to support reactive metal-oxo or metal-peroxo species (for an example, see: M. S. Chen, M. C. White Science 2007, 318, 783). Curci and coworkers have used dioxiranes - strained, electrophilic heterocycles - for the oxygenation of saturated hydrocarbons (Acc. Chem. Res. 2006, 39, 1). In contrast the Du Bois group employs in situ generated oxaziridines $\mathbf{2}$ for the same purpose. Compared to their first-generation process the advanced method not only simplifies the experimental protocol by avoiding $\mathrm{Ar}_{2} \mathrm{Se}_{2}$ as co-catalyst but also has a significantly broader substrate scope. 